\title{
Shear Test of Variable Depth RC Beams with Inflection Point
}

\author{
Kaiqi Zheng ${ }^{1,2, *}$, Michael Kuwornu ${ }^{2}$, and Zhao Liu ${ }^{1}$ \\ ${ }^{1}$ Southeast University, Key laboratory of concrete and pre-stressed concrete structures of Ministry of Education, 210096 Nanjing, China \\ ${ }^{2}$ Nanjing Forestry University, College of Civil Engineering, 210037 Nanjing, China
}

\begin{abstract}
Variable depth continuous box girder bridges are widely used recently. However, the influence mechanism of the inflection point and variable depth on the shear behaviour of this type bridge has not been fully understood. In this paper, reinforced concrete (RC) beams with constant depth and variable depth were designed as constrained beams to create the inflection point. Firstly, a theoretical uncoupling theory for shear and flexural deformation of variable depth beam was derived. Then, the after cracking shear deformations and the ultimate failure loads were measured. Further, preliminary reveal of the influence mechanism of inflection point on the shear capacity was attempted. Finally, the development of aftercracking shear strain and the degradation of shear stiffness were studied. The formation of first critical diagonal cracks and yielding of stirrup are the turning points of shear stiffness. Increasing stirrup ratio provides more apparent enhancement for residual shear stiffness, which means more effective inhibition on shear deformation.
\end{abstract}

\section{Introduction}

With the continuous development of concrete bridges, large span variable depth continuous box girder bridges have been widely used in practice. Compared with ordinary simply supported concrete bridges, variable depth and inflection point are two special characteristics that further increases the difficulty of the complicated shear analysis of RC beams.

The behavior of variable depth beams with regard to shear may differ from that of constant depth members for the reason that the inclination of the main internal tension or compression chord in a tapered beam provides a vertical component potentially capable of resisting shear ${ }^{[1]}$. Also, the existence of inflection point, in a certain extent, will decreases the capacity to resist shear $^{[2]}$. Moreover, Literature research related to shear in RC beams mainly focused on shear capacity ${ }^{[3-4]}$. Though, as early as 1960 s, The Stuttgart shear tests indicated that for concrete beams with $l / h$ ranging from 5 to 12 , shear deformation were between 0.2 and 3.0 times that of the flexural deformation ${ }^{[5]}$. Until recently, some investigations on shear deformation confirmed that shear contribution to the total deflection in the diagonally cracked RC beam is significant ${ }^{[6-7]}$. However, there is still lack of such kind of experimental research in literature.

As continued variable depth concrete bridges are frequently used in practice, it is necessary to give a detailed investigation on the shear behavior of variable depth beams combined with inflection point. This paper aims to reveal that how variable depth and inflection point affect the mechanism of shear transfer and to extend the generally used shear design approach to beams with non-prismatic section or beams with inflection point. Six carefully designed RC beams were tested to fail in shear. The effect of variable depth and effect of inflection point on beam shear capacity were experimentally investigated and discussed. On this basis, modified shear capacity of this type beams was proposed and verified.

\section{Test Program}

\subsection{Specimens and Material Properties}

Six specimens with I shaped cross-section belonging to two series were designed in the experimental program. Each series consists a reference beam with constant depth $h=800 \mathrm{~mm}$, and two beams with variable depth $(h$ varies from $800 \mathrm{~mm}$ to $400 \mathrm{~mm}$ ). All specimens with the same beam length $5.4 \mathrm{~m}$ were simply supported with a single cantilever (Fig. 1). Specially, for variable depth specimen, the bottom surface has a quadric parabolic alignment, while the top surface keeps horizontal.

In all six specimens, D25 bars and D12 bars with a total cross section area $3624 \mathrm{~mm}^{2}$ and average yield strength of $497 \mathrm{MPa}$ were used as longitudinal bars, which were arranged symmetrically in top and bottom flanges.D8 round bars (two legs) with average yield strength of $326 \mathrm{MPa}$ were arranged as stirrups at the spacing of $200 \mathrm{~mm}\left(\rho_{\mathrm{v}}=0.5 \%\right)$ or $250 \mathrm{~mm}\left(\rho_{\mathrm{v}}=0.4 \%\right)$ for deferent beams along the whole span. The average concrete cylinder strengths were $39.0 \mathrm{MPa}$ and $36.0 \mathrm{MPa}$ for series I and II, respectively. It must be mentioned that excessive longitudinal reinforcement are arranged to prevent early flexural failure. Table 1 summarizes and

Corresponding author: k.zheng@njfu.edu.cn 
illustrates the details of the tested beams, including the dimension and reinforcing bars arrangement.

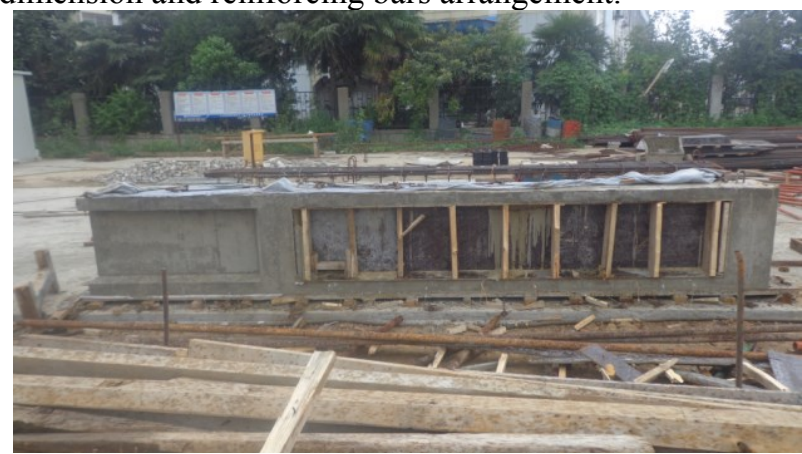

(a)

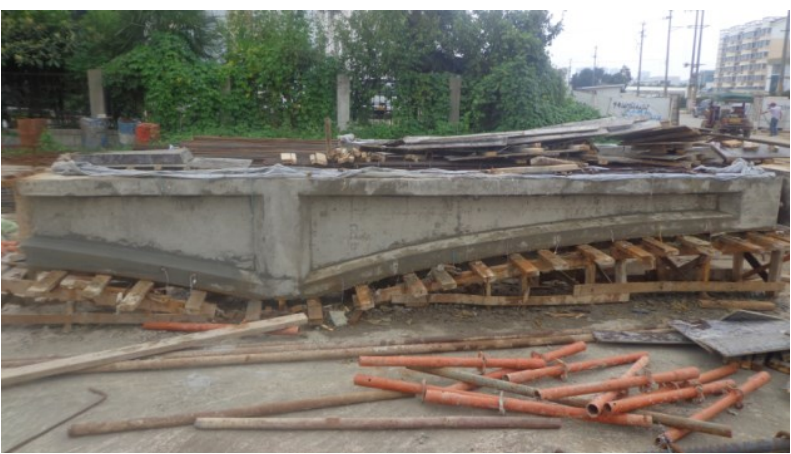

(b)

Fig. 1. Specimens: (a) constant depth, (b) variable depth

Table 1. Specimen details and material properties.

\begin{tabular}{|c|c|c|c|c|c|c|c|c|c|}
\hline \multirow[t]{2}{*}{ Series } & \multirow[t]{2}{*}{ Specimen } & \multirow[t]{2}{*}{$b_{\mathrm{w}}(\mathrm{mm})$} & \multirow[t]{2}{*}{$h(\mathrm{~mm})$} & \multirow[t]{2}{*}{$f_{\mathrm{c}}^{\prime}(\mathrm{MPa})$} & \multicolumn{2}{|c|}{ Shear reinforcement } & \multicolumn{3}{|c|}{ Flexural Reinforcements } \\
\hline & & & & & Arrangement & $f_{\mathrm{yv}}(\mathrm{MPa})$ & Top Flange & $\begin{array}{l}\text { Bottom } \\
\text { flange }\end{array}$ & $f_{\mathrm{y}}(\mathrm{MPa})$ \\
\hline \multirow{3}{*}{ I } & $\mathrm{BC} 1$ & 100 & 800 & 39.0 & D8(2)@200 & 326 & $6 \mathrm{D} 25+6 \mathrm{D} 12$ & $6 \mathrm{D} 25+6 \mathrm{D} 12$ & 497 \\
\hline & BV1 & 100 & $400 \sim 800$ & 39.0 & D8(2)@200 & 326 & $6 \mathrm{D} 25+6 \mathrm{D} 12$ & $6 \mathrm{D} 25+6 \mathrm{D} 12$ & 497 \\
\hline & BV2 & 100 & $400 \sim 800$ & 39.0 & D8(2)@250 & 326 & $6 \mathrm{D} 25+6 \mathrm{D} 12$ & $6 \mathrm{D} 25+6 \mathrm{D} 12$ & 497 \\
\hline \multirow{3}{*}{ II } & $\mathrm{BC} 2$ & 100 & 800 & 36.0 & D8(2)@250 & 326 & $6 \mathrm{D} 25+6 \mathrm{D} 12$ & $6 \mathrm{D} 25+6 \mathrm{D} 12$ & 497 \\
\hline & BV3 & 100 & $400 \sim 800$ & 36.0 & D8(2)@200 & 326 & $6 \mathrm{D} 25+6 \mathrm{D} 12$ & $6 \mathrm{D} 25+6 \mathrm{D} 12$ & 497 \\
\hline & BV4 & 100 & $400 \sim 800$ & 36.0 & D8(2)@250 & 326 & $6 \mathrm{D} 25+6 \mathrm{D} 12$ & $6 \mathrm{D} 25+6 \mathrm{D} 12$ & 497 \\
\hline
\end{tabular}

$b_{\mathrm{w}}$ : web width of beam; $h$ : height of beam; $f_{\mathrm{c}}^{\prime}$ : compressive strength of concrete; $f_{\mathrm{yv}}$ : yield strength of shear reinforcement; $f_{\mathrm{y}}:$ yield strength of flexural reinforcement.

(a)

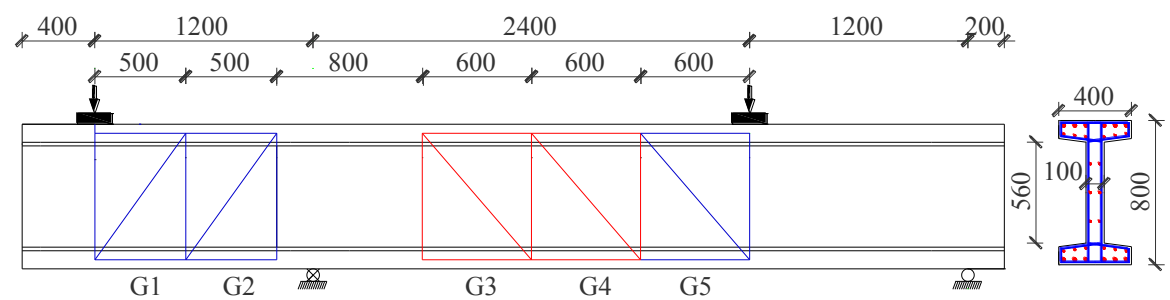

(b)

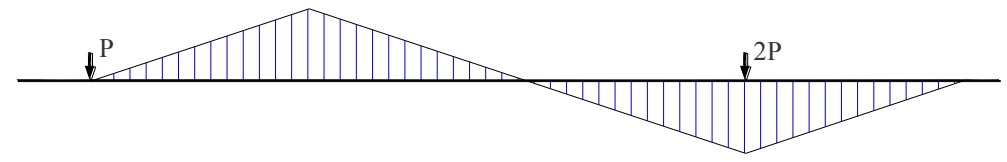

$P$

(c)

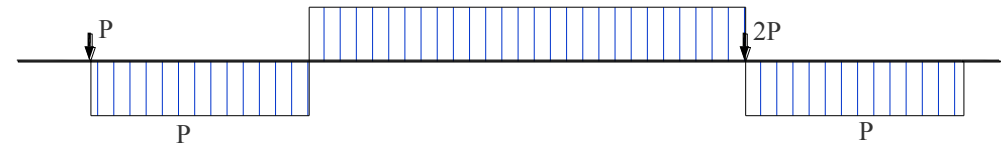

Fig. 2. Geometry, instrumentation and loading for (BC1, $\mathrm{BC} 2)$ : (a) Geometry(unit: mm), (b)M diagram, (c) $\mathrm{Q}$ diagram 
(a)

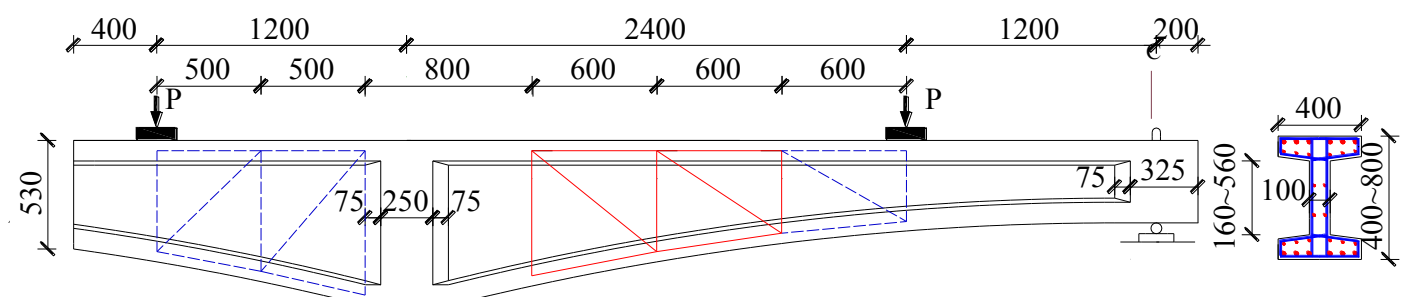

G1

$\mathrm{G} 2$

G3

G4

G5

$1.2 \mathrm{P}$

(b)

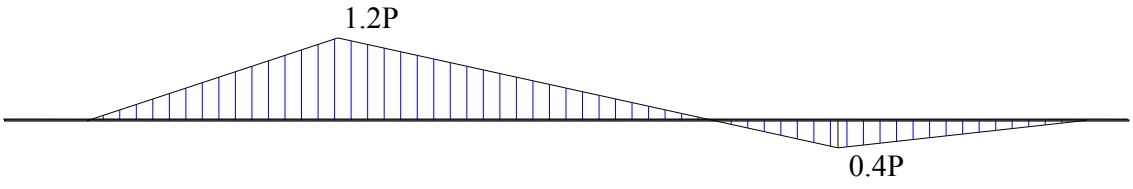

(c)

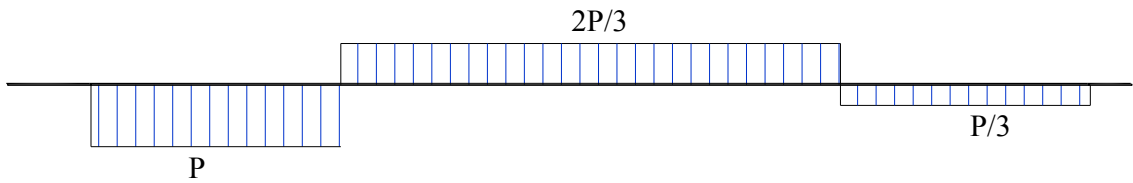

Fig. 3. Geometry, instrumentation and loading for variable depth beams (BV1, BV2, BV3, BV4): (a) Geometry(unit: mm), (b)M diagram, (c) Q diagram

\subsection{Test setup and instrumentation}

During the loading phase, two synchronous loading cells are assigned to the cantilever end and within the span respectively. Geometry and loading setup for the specimens are depicted in Fig. 2 and 3.

To ensure that the continuous observation of strain and deformation in the web zone after diagonal cracking, Five specifically designed measuring grids G1 $\sim$ G5 are arranged on one side of the beams (Fig. 2 and 3). This strain measuring grids are composed by 5 mechanical strain gauges with large base length. The strain gauge contains a dial gauge, a gauge handle and an extension rod, and is fixed by expansion bolt at its ends. Shear deformation of beam segment before and after diagonal cracking will be measured and calculated by proposed uncoupling theory in the next section.

During the tests, the beam deflection was measured using ten displacement transducers at each node of all measuring grids and supporting points. Gauges were attached on the surface of stirrups and longitudinal reinforcement at various locations for strain monitoring and yielding judgments during the loading process.

In addition, the crack propagation on the surface of test shear span during the loading test was captured in pictures.

\section{Deformation uncoupling theory}

As the beam was subjected to combined action of shear and bending moment, the corresponding shear and flexural deformation are coupled with each other. To strip flexural deformation off from the total deformation, a refined uncouple theory was needed.

\subsection{Basic assumption}

To uncouple shear and flexural deformation, the integral relationship between strain and deformation was employed in a previous Chinese article by the authors ${ }^{[8]}$. For ease of understanding and application, the detailed process of derivation was introduced here. As we known, Flexural deformation can be expressed as the double integration of curvature $\rho$ along the length $l$, and shear deformation can be expressed as the integration of shear strain $\gamma_{x y}$ along the length $l$ (Eq. (1) and (2)). Elastic theory also gives basic definitions for curvature $\rho$ and shear strain $\gamma_{x y}$ based on rectangle element (Fig. 4).

$$
\begin{aligned}
& f_{b}=\int_{0}^{l}\left(\int_{0}^{l} \rho d x\right) d x \\
& f_{s}=\int \gamma_{x y} d x
\end{aligned}
$$
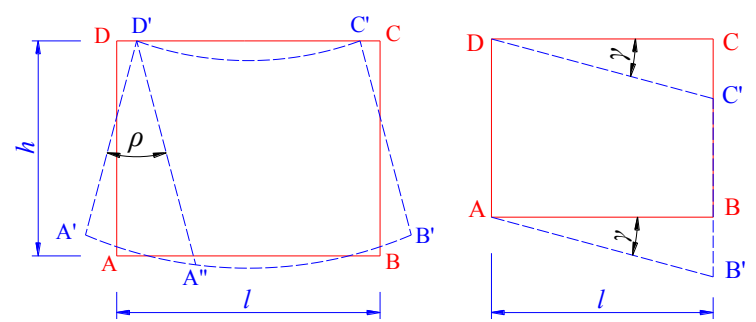

Fig. 4. Definition for curvature $\rho$ and shear strain $\gamma_{x y}$

As the node's displacement of the grid was recorded, strain is worked out through their partial differential relationship showed below. 


$$
\boldsymbol{\varepsilon}=\left(\begin{array}{c}
\varepsilon_{x} \\
\varepsilon_{y} \\
\gamma_{x y}
\end{array}\right)=\mathbf{A}^{\mathrm{T}} \mathbf{d}=\left(\begin{array}{ccc}
\frac{\partial}{\partial x} & 0 & \frac{\partial}{\partial y} \\
0 & \frac{\partial}{\partial y} & \frac{\partial}{\partial x}
\end{array}\right)^{T}\left(\begin{array}{l}
u \\
v
\end{array}\right)
$$

Where, $\boldsymbol{\varepsilon}$ and $\mathbf{d}$ are the strain matrix and displacement matrix of the grid respectively, $\mathbf{A}$ is the partial differential operator matrix.

For a square or rectangular measuring grid, mean strain can be used in Eq. (1) and (2), which still keeps high precision. Mean curvature $\rho$ is expressed as the ratio of strain difference of lower and upper chord to the grid height. And shear strain $\gamma_{x y}$ can be easily calculated out according to the three-dimensional rosette strain[8].

However, for irregular quadrilateral grid, those basic definitions above are not accurate, and may cause excessive deviation. To overcome this trouble, the isoparametric concept from finite element method was adopted.

It is assumed that strain and displacement has a linear distribution in the quadrilateral grid element (namely, the inherit element). To describe the strain distribution within the grid, a 4 nodes square element (namely, the parent element) and its corresponding shape function $\mathbf{N}$ are established (Eq. (4)). Inherit element has two coordinate system, its own global coordinate system $(x$, $y$ ), and the local coordinate system $(\xi, \eta)$ inherited from the parent element (Fig. 5).

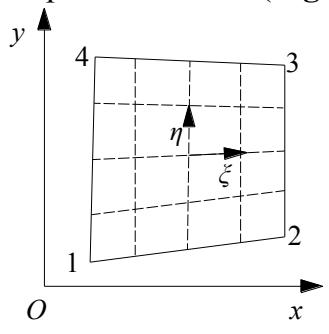

(a) inherit element

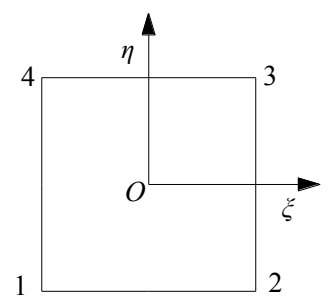

(b) parent element
Fig. 5. 4 nodes iso-parametric element

For 4 nodes square parent element, it's shape function can be given as follows:

$$
N_{i}=\left(1+\xi_{i} \xi\right)\left(1+\eta_{i} \eta\right) / 4 \quad(i=1,2,3,4)
$$

Where, $\xi, \eta$ are the local coordinates of arbitrary point in the parent element, and $\xi_{i}, \eta_{i}$ the local coordinates of node $i$ of the parent element.

Geometry and displacement of the grid element can be depicted by interpolation with the parent element's shape function $\mathbf{N}$. Known the node coordinates and node displacements of the grid element, the coordinates and displacements of any point within the grid element can be described as follows:

$$
\begin{aligned}
\mathbf{x} & =\left(\begin{array}{ll}
x & y
\end{array}\right)^{\mathrm{T}}=\mathbf{N} \mathbf{x}^{\mathbf{e}} \\
& =\left(\begin{array}{lllll}
N_{1} \mathbf{I}_{2} & N_{2} \mathbf{I}_{2} & N_{3} \mathbf{I}_{2} & N_{4} \mathbf{I}_{2}
\end{array}\right)\left(\begin{array}{llll}
x_{1} & y_{1} & \cdots
\end{array}\right. \\
\mathbf{d} & =\left(\begin{array}{ll}
u & v
\end{array}\right)^{\mathrm{T}}=\mathbf{N} \mathbf{N d}^{\mathrm{e}} \\
& =\left(\begin{array}{lllll}
N_{1} \mathbf{I}_{2} & N_{2} \mathbf{I}_{2} & N_{3} \mathbf{I}_{2} & N_{4} \mathbf{I}_{2}
\end{array}\right)\left(\begin{array}{lllll}
u_{1} & v_{1} & \cdots
\end{array}\right.
\end{aligned}
$$

Where, $\mathbf{x}$ and $\mathbf{d}$ are the coordinate matrix and displacement matrix of the quadrilateral grid element respectively, $\mathbf{x}^{\mathrm{e}}$ and $\mathbf{d}^{\mathrm{e}}$ are the node coordinate matrix and node displacement matrix of the grid element respectively, $\mathbf{I}_{2}$ is a second-order identity matrix, $\mathbf{N}$ is the shape function matrix.

As the grid displacement $\mathbf{d}$ is described with the shape function $\mathbf{N}$, which is expressed by the local coordinate system, the differential formula of displacement to strain must be transformed from global coordinates to local coordinates. Based on the differential rules of composite functions and matrix operations, partial differential operators can be obtained:

$$
\left(\begin{array}{c}
\frac{\partial}{\partial x} \\
\frac{\partial}{\partial y}
\end{array}\right)=\frac{1}{\operatorname{det} \mathbf{J}}\left(\begin{array}{cc}
\sum_{i} y_{i} \frac{\partial N_{i}}{\partial \eta} & -\sum_{i} y_{i} \frac{\partial N_{i}}{\partial \xi} \\
-\sum_{i} x_{i} \frac{\partial N_{i}}{\partial \eta} & \sum_{i} x_{i} \frac{\partial N_{i}}{\partial \xi}
\end{array}\right)\left(\begin{array}{c}
\frac{\partial}{\partial \xi} \\
\frac{\partial}{\partial \eta}
\end{array}\right)
$$

Where, det $\mathbf{J}$ is the determinant of Jacobian matrix,

$$
\operatorname{det} \mathbf{J}=\left(\sum_{i} x_{i} \frac{\partial N_{i}}{\partial \xi}\right)\left(\sum_{i} y_{i} \frac{\partial N_{i}}{\partial \eta}\right)-\left(\sum_{i} y_{i} \frac{\partial N_{i}}{\partial \xi}\right)\left(\sum_{i} x_{i} \frac{\partial N_{i}}{\partial \eta}\right)
$$

According to the partial differential relationship between the strain and displacement, shear strain $\gamma_{x y}$ can be expressed as:

$$
\begin{gathered}
\varepsilon_{x}(\xi, \eta)=\frac{1}{\operatorname{det} \mathbf{J}}\left(\sum_{i} y_{i} \frac{\partial N_{i}}{\partial \eta} \cdot \sum_{i} u_{i} \frac{\partial N_{i}}{\partial \xi}-\sum_{i} y_{i} \frac{\partial N_{i}}{\partial \xi} \cdot \sum_{i} u_{i} \frac{\partial N_{i}}{\partial \eta}\right) \\
\varepsilon_{y}(\xi, \eta)=\frac{1}{\operatorname{det} \mathbf{J}}\left(\sum_{i} x_{i} \frac{\partial N_{i}}{\partial \xi} \cdot \sum_{i} v_{i} \frac{\partial N_{i}}{\partial \eta}-\sum_{i} x_{i} \frac{\partial N_{i}}{\partial \eta} \cdot \sum_{i} v_{i} \frac{\partial N_{i}}{\partial \xi}\right) \\
\gamma_{x y}(\xi, \eta)=\frac{1}{\operatorname{det} \mathbf{J}}\left(\sum_{i} x_{i} \frac{\partial N_{i}}{\partial \xi} \cdot \sum_{i} u_{i} \frac{\partial N_{i}}{\partial \eta}-\sum_{i} x_{i} \frac{\partial N_{i}}{\partial \eta} \cdot \sum_{i} u_{i} \frac{\partial N_{i}}{\partial \xi}\right)+\frac{1}{\operatorname{det} \mathbf{J}}\left(\sum_{i} y_{i} \frac{\partial N_{i}}{\partial \eta} \cdot \sum_{i} v_{i} \frac{\partial N_{i}}{\partial \xi}-\sum_{i} y_{i} \frac{\partial N_{i}}{\partial \xi} \cdot \sum_{i} v_{i} \frac{\partial N_{i}}{\partial \eta}\right)
\end{gathered}
$$

\subsection{Segment shear deformation \& flexural deformation}

As strain is obtained by the analysis above, flexural deformation of the grid can be calculated with Eq. (1).
To avoid the integral procedure, some simplifications are made to Eq. (1). From the linear strain distribution assumption, average curvature $\rho$ is employed, which is the ratio of strain difference of upper and lower chord to the grid height $h$. Also with the help of iso-parametric concept, we get: 


$$
\begin{aligned}
\rho & =\frac{\varepsilon_{x u}-\varepsilon_{x b}}{h}=\left.\frac{1}{2 h} \int_{-1}^{1} \varepsilon_{x}(\xi, \eta) d \xi\right|_{\eta=-1} ^{\eta=1}=\text { const } \\
f_{b} & =\int_{0}^{l}\left(\int_{0}^{l} \rho d x\right) d x \\
& =\left.\frac{l^{2}}{4 h} \int_{-1}^{1} \varepsilon_{x}(\xi, \eta) d \xi\right|_{\eta=-1} ^{\eta=1}=\left.\left.\frac{l^{2}}{2 h} \varepsilon_{x}\right|_{\xi=0}\right|_{\eta=-1} ^{\eta=1}
\end{aligned}
$$

Correspondingly, the length $l$ and height $h$ of the grid can be deduced by geometrical relationship:

$$
\begin{aligned}
& l=\frac{1}{2} \sqrt{\left(x_{2}+x_{3}-x_{1}-x_{4}\right)^{2}+\left(y_{2}+y_{3}-y_{1}-y_{4}\right)^{2}} \\
& h=\frac{1}{2} \sqrt{\left(x_{1}+x_{2}-x_{3}-x_{4}\right)^{2}+\left(y_{1}+y_{2}-y_{3}-y_{4}\right)^{2}}
\end{aligned}
$$

Simplification for Eq. (2) for shear deformation was performed analogically. The vertical shear deformation of the grid can be represented by the multiply of average shear strain $\bar{\gamma}$ with its length $l$ :

$$
f_{s}=\int \gamma_{x y} d x=\int \bar{\gamma} d x=\left.\gamma_{x y}(\xi, \eta)\right|_{\xi=0, \eta=0} \cdot l
$$

For a measuring grid on specimen, the corresponding segment flexural and shear deformation can be obtained according to Eq. (12) and Eq. (15) separately.

\section{Test Results and Discussions}

\subsection{Failure mode}

During the loading process, the flexural cracking propagation was very slow, and was limited to the flanges. Meanwhile, shear cracks developed quickly from the middle of beam web. The critical diagonal crack propagated from middle of web, and penetrated to top and bottom flanges gradually. Finally, it turns out to be a web shear failure at the inflection point, the web concrete is collapsed after stirrup yielding (Fig. 6).
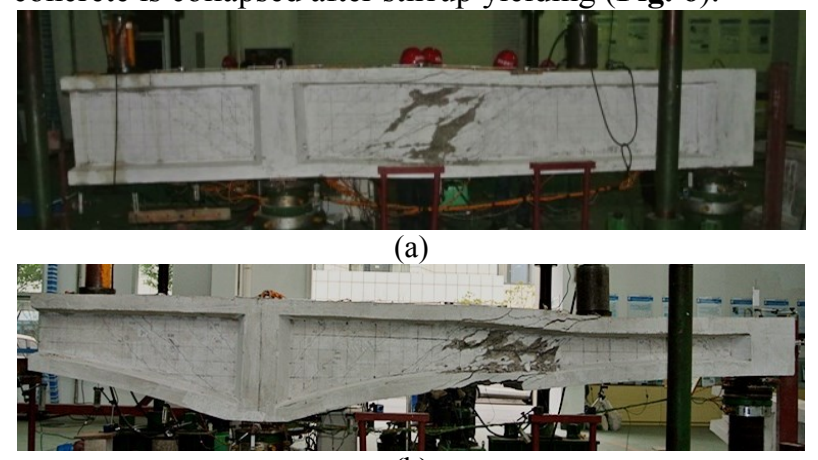

(b)

Fig. 6. Typical failure modes of specimens: $(a) \mathrm{BC} 2 ;(b) \mathrm{BV} 3$

The sequences of diagonal crack propagating, stirrup yielding and final failure for each measuring segment are listed in Table 2. The first shear crack always develops from the segment with the maximum shear stress and flexural stress. While the stirrup yielding and shear failure are all concentrated in the vicinity of the segment with minimum generalized shear span ratio, namely the inflection point.

Table 2. Sequences of diagonal crack propagating, stirrup yielding and final failure for each segment

\begin{tabular}{ccccccc}
\hline \multirow{2}{*}{ Specimen } & $\begin{array}{c}\text { Segment shear } \\
\text { (kN) }\end{array}$ & \multicolumn{5}{c}{ Measured segment } \\
\cline { 3 - 7 } BC1 & $Q_{\text {cr }}$ & 240 & 150 & 170 & 170 & 220 \\
& $Q_{\mathrm{y}}$ & 320 & 320 & 220 & 220 & - \\
& $Q_{\mathrm{u}}$ & - & - & 400 & 400 & - \\
\hline \multirow{3}{*}{ BV1 } & $Q_{\text {cr }}$ & 180 & 180 & 160 & 133 & 120 \\
& $Q_{\mathrm{y}}$ & 300 & 280 & 187 & 200 & 200 \\
& $Q_{\mathrm{u}}$ & - & - & - & 307 & 307 \\
\hline \multirow{3}{*}{ BV2 } & $Q_{\text {cr }}$ & 210 & 210 & 133 & 140 & 140 \\
& $Q_{\mathrm{y}}$ & - & - & 200 & 213 & 213 \\
& $Q_{\mathrm{u}}$ & - & - & - & 297 & 297 \\
\hline \multirow{3}{*}{ BC2 } & $Q_{\text {cr }}$ & 220 & 170 & 180 & 200 & 200 \\
& $Q_{\mathrm{y}}$ & 340 & 340 & 240 & 240 & - \\
& $Q_{\mathrm{u}}$ & - & - & 350 & 350 & - \\
\hline \multirow{3}{*}{ BV3 } & $Q_{\mathrm{cr}}$ & 160 & 160 & 147 & 107 & 93 \\
& $Q_{\mathrm{y}}$ & - & - & 200 & 200 & 200 \\
& $Q_{\mathrm{u}}$ & - & - & - & 293 & 293 \\
\hline \multirow{3}{*}{ BV4 } & $Q_{\mathrm{cr}}$ & 140 & 140 & 120 & 127 & 127 \\
& $Q_{\mathrm{y}}$ & 280 & 280 & 180 & 200 & 200 \\
& $Q_{\mathrm{u}}$ & - & - & - & 300 & 300 \\
\hline
\end{tabular}

$Q_{\mathrm{cr}}$ : Shear force for first diagonal crack; $Q_{\mathrm{y}}$ : Shear force for stirrup yielding; $Q_{\mathrm{u}}$ : Shear force for web failure; “-”: Not reached.

\subsection{Effect of inflection point on shear capacity}

As all six specimens failed with web shear failure in the vicinity of inflection point, it is necessary to make sure that the currently used shear design methods are applicable for such members. The test results are compared with predictions by three popularly shear model $^{[9]}$ : 45 degree truss model, variable angle truss model (VATM), and modified compression field theory(MCFT). The main results are shown in Table 3.

\begin{tabular}{|c|c|c|c|c|c|c|c|}
\hline No. & $\begin{array}{c}V_{\text {test }} \\
(\mathrm{kN})\end{array}$ & $\begin{array}{c}V_{\mathrm{He}} \\
(\mathrm{kN})\end{array}$ & $\begin{array}{l}V_{\mathrm{ACI}} \\
(\mathrm{kN})\end{array}$ & $\begin{array}{l}V_{\mathrm{AAS}} \\
(\mathrm{kN})\end{array}$ & $\frac{V_{\text {test }}}{V_{\mathrm{ACI}}}$ & $\frac{V_{\text {test }}}{V_{\mathrm{He}}}$ & $\frac{V_{\text {test }}}{V_{\mathrm{AAS}}}$ \\
\hline $\mathrm{BC} 1$ & 220 & 289 & 203 & 331 & 1.09 & 0.76 & 0.66 \\
\hline BV1 & 200 & 175 & 123 & 203 & 1.63 & 1.14 & 0.99 \\
\hline BV2 & 213 & 150 & 108 & 178 & 1.98 & 1.42 & 1.20 \\
\hline $\mathrm{BC} 2$ & 240 & 242 & 175 & 285 & 1.37 & 0.99 & 0.84 \\
\hline BV3 & 200 & 171 & 121 & 199 & 1.66 & 1.17 & 1.00 \\
\hline BV4 & 200 & 146 & 106 & 176 & 1.89 & 1.37 & 1.14 \\
\hline \multirow{2}{*}{\multicolumn{5}{|c|}{$\begin{array}{r}\text { Mean } \\
\text { Coefficient of Variation }\end{array}$}} & 1.60 & 1.14 & 0.97 \\
\hline & & & & & 0.21 & 0.21 & 0.20 \\
\hline
\end{tabular}
The $V_{\text {test }}$ used in Table 3 is the shear force at stirrup yielding. After the yielding of the stirrups, a considerable load increase is possible up to the shear failure, which is not discussed in this paper.

Table 3. Verification with three shear design method

As can be seen in Table $\mathbf{3}$, all three methods give more conservative prediction for variable depth members than constant members, which indicates that the inclined chord of the variable depth members do give some contribution for their shear capacity.

The ACI method ${ }^{[10]}$ based on 45 degree truss model gives conservative predictions both for constant depth 
members and variable depth members. While the He's method $^{[11]}$ based on VATM and the AASHTO method ${ }^{[12]}$ based on MCFT tender to give unsafe predictions for constant members. This fact gives evidence that the shear transfer mechanism for beam with inflection point is very different from a normal beam. The change from positive moment to negative moment tends to tear the upper and lower chord away from each other. This tendency increases the concrete strain in longitudinal direction and may further "soften" the inclined concrete struts divided by the diagonal cracks and lead to their early crush.

\subsection{Shear strain after shear cracking}

Based on the approach proposed in section 3, shear, strain can be measured and calculated separately. As grid G3 \& G4 are located in the vicinity of inlection point, the flexural deformaiton are very small while shear deformation accounts for the majority. The shear strain curves in Grid G3 \& G4 are drawn in Fig.7.

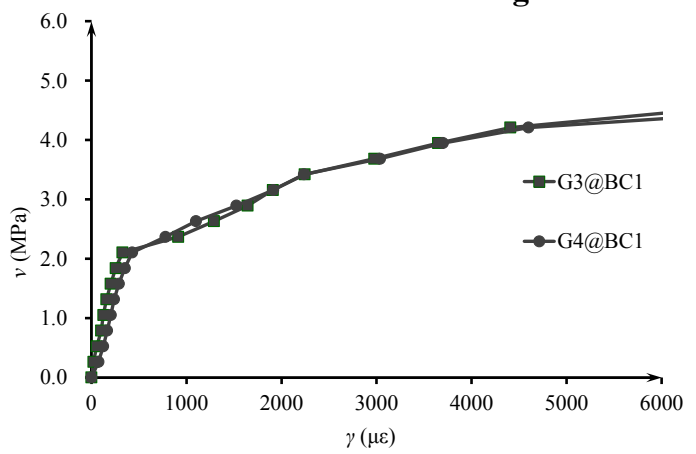

(a) $\mathrm{BC} 1$

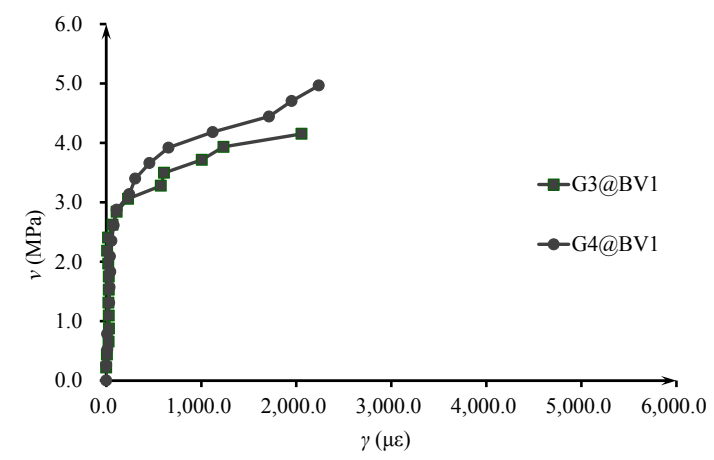

(b) BV1

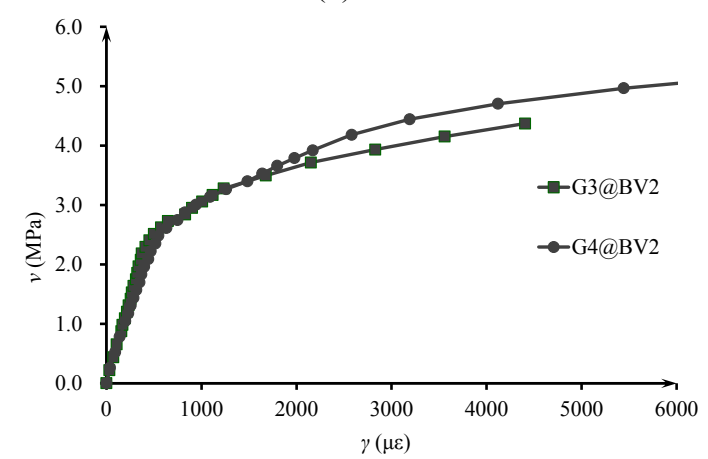

(c) BV2

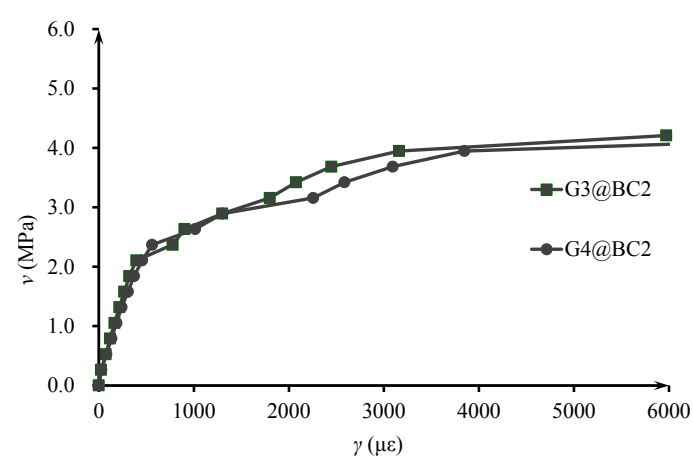

(d) $\mathrm{BC} 2$

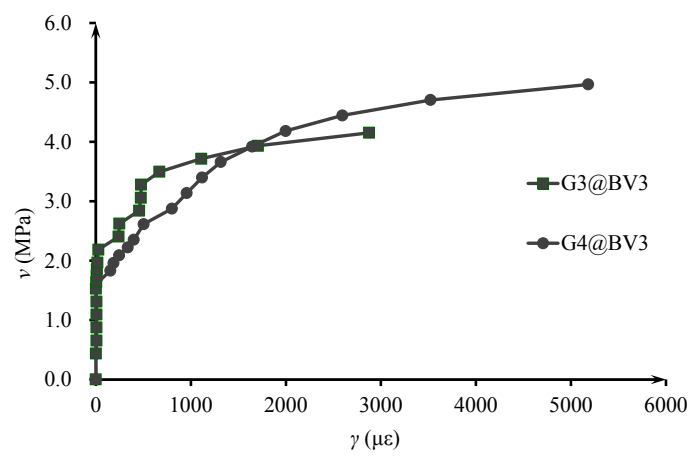

(e) BV3

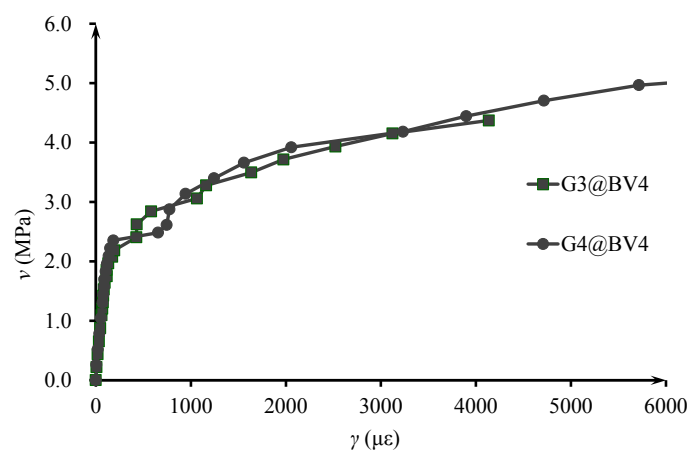

(f) BV4

Fig. 7. Shear stress-strain curves for gridG3 \&G4

As is shown in Fig. $7(a)$ to $\mathbf{7}(f)$, the shear strain curve has a turn point when the first shear crack occurs(cracking stress is about $2.0 \mathrm{MPa}$ ), and then increases quickly. It shows a "yield plateau" after the stirrup yielding, which means severe degradation of shear stiffness. When the ultimate load is reached, shear strain increases to dozens of times that of uncracked phase. Throughout the loading process, flexural deformation keeps within a small range, and has no obvious turns. First shear crack and stirrup yielding are the turn points of beam shear strain curve.

\subsection{Degradation of shear stiffness after shear cracking}

Shear stiffness is the most important indicator for shear deformation evaluation. Shear stiffness $\mathrm{K}_{\mathrm{e}}$ of a section can be expressed as $G A_{\mathrm{w}}$ ( $\mathrm{G}$ is the concrete elastic shear modulus; $A_{\mathrm{w}}=b_{\mathrm{w}} d$, where $b_{\mathrm{w}}$ is web width, $d$ is the effective depth)during the elastic phase. Considering the Poisson's ratio, it became $0.42 E A_{\mathrm{w}}$ ( $E$ is the concrete elastic modulus). However, after diagonal cracking, 
structural continuity was damaged and Poisson effect no longer existed, elastic shear stiffness lost its basement.

Generally, nominal shear stress $\tau$ can be considered as the ratio of shear force $P$ to the effective shear area $A_{\mathrm{w}}$. As the average shear strain is measured, based on the constitution relationship of stress and strain, an equivalent shear stiffness $K_{\text {eq }}$ can be defined as follows:

$$
K_{\mathrm{eq}}=\frac{\tau}{\bar{\gamma}} A_{w}=\frac{P}{\bar{\gamma}}
$$

In order to study the variation of shear stiffness during diagonal cracking propagation, shear stiffness reduction factor $\beta$ is defined as the ratio of the equivalent shear stiffness $K_{\text {eq }}$ to the elastic shear stiffness $\mathrm{K}_{\mathrm{e}}$,

$$
\beta=K_{\text {eq }} / K_{\mathrm{e}}
$$

Considering that shear strain is very small during elastic stage, take $\beta=1$ when calculated $\beta>1$. Shear stiffness reduction factor varying with load was drawn in Fig. 8.

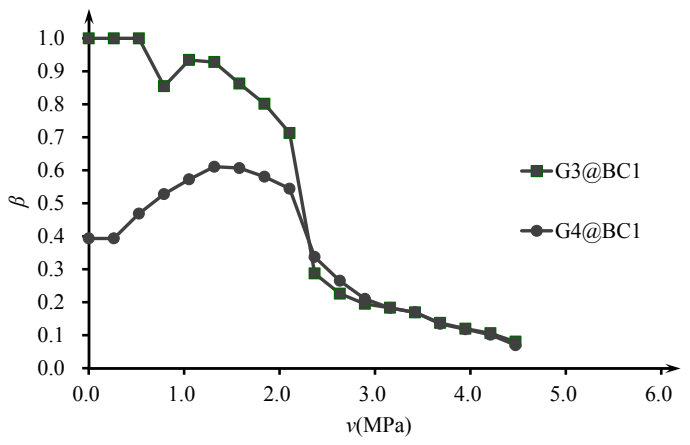

(a) $\mathrm{BC} 1$

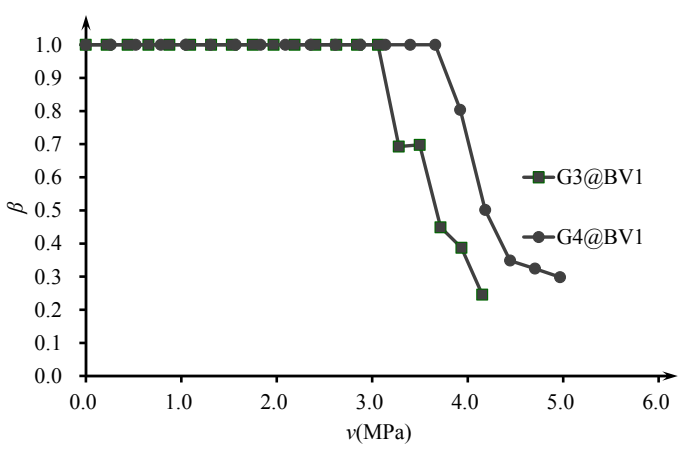

(b) BV1

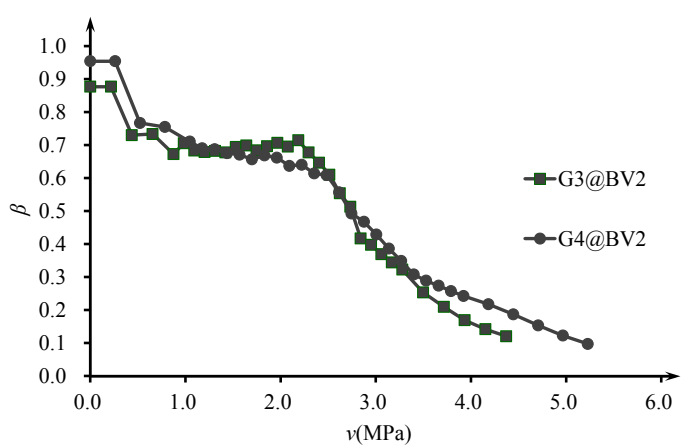

(c) BV2

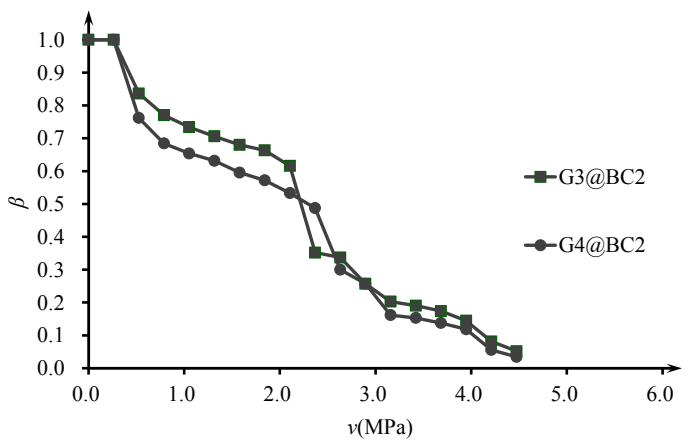

(d) $\mathrm{BC} 2$

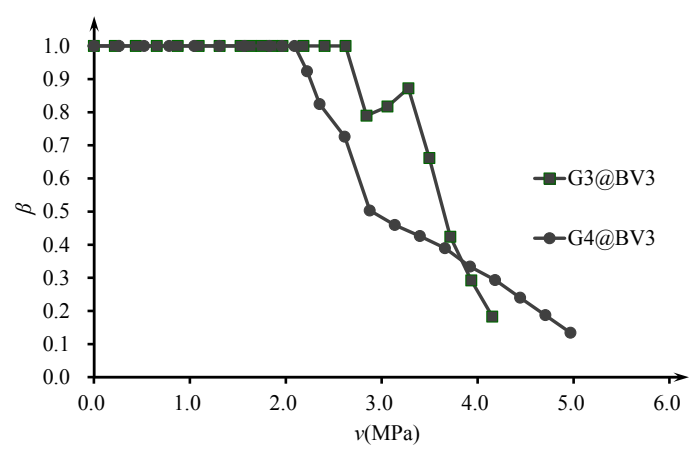

(e) BV3

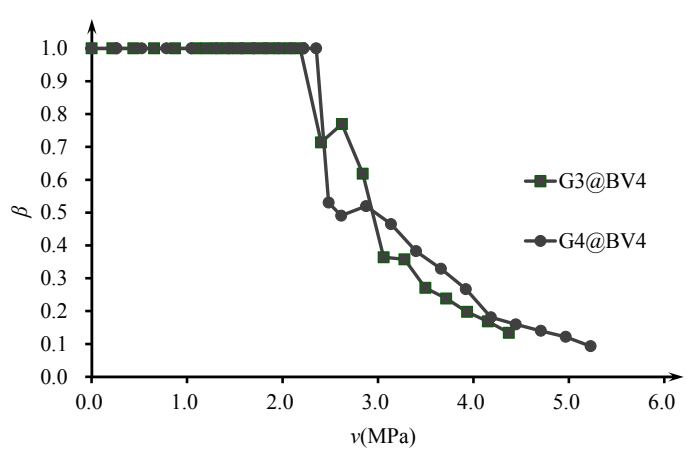

(f) BV4

Fig. 8. Variation of shear stiffness reduction factor $\beta$ with shear stress

As can be seen, the characteristic loads of two obvious stiffness reduction coincide with the first diagonal crack concrete shear stress $(2.0 \mathrm{MPa})$ and stirrup yielding concrete shear stress $(4.0 \mathrm{MPa})$ exactly. it can be inferred that the shear stiffness reduction factor $\beta$ can reflect the impact of the web diagonal cracking and stirrup yielding on the shear stiffness.

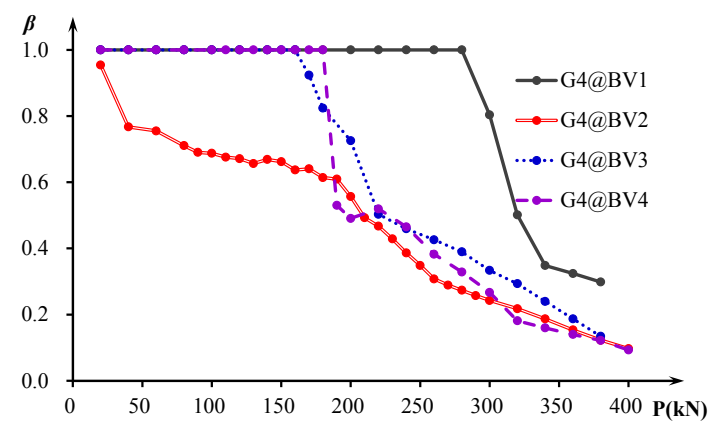

Fig. 9. Shear stiffness reduction factor $\beta$ for grid $\mathrm{G} 4$

Shear stiffness degradation of specimen BV1 to BV4 are compared in Fig.9. As the stirrup ratios are kept on lower levels in these specimen series, first diagonal 
cracking induced shear stiffness degradation accounts a lot. Two main parameters, concrete strength and stirrup ratio, are studied. Raising the concrete strength and stirrup ratio can improve the residual shear stiffness after shear diagonal cracking. Before stirrup yielding, increasing stirrup ratio provides more apparent enhancement for residual shear stiffness, which means more effective inhibition on shear deformation (Fig. 8 (b)). After the specimen diagonal cracking, shear stiffness remains $30 \%$ to $40 \%$, while after stirrup yielding it reduces to about $10 \%$.

\section{Summary and Conclusions}

The purpose of the current study is to obtain detailed information on shear cracking behavior in variable depth RC beams with inflection point. The experiment investigates the effect of various parameters such as variable depth, inflection point, concrete strength, and stirrup spacing on the after-cracking behavior: shear capacity and shear stiffness. Based on the experimental results, the following conclusions can be derived, which are useful information for the development of a rational shear capacity and shear stiffness prediction method in existing design codes.

1) The test results indicates that the beam inflection point is the weak area. Shear failure always initiates from the web stirrup yielding and web concrete crush. The tested shear capacity is compared to three popular shear model, which shows that VATM and MCFT tender to give unsafe predictions for constant members. The conversion of beam moment increases the longitudinal strain and "softens" the inclined concrete strut, which consequently decreases the shear capacity.

2) With the arrangement of self-designed strain measuring grids on the specimens, continuous strain measurements is achieved during the whole cracking process. A corresponding theoretical uncoupling approach for shear deformation measurement of variable depth beam is proposed. With the help of isoparametric transformation, the shear deformation of the quadrilateral grid is measured and calculated based on its basic definitions. This approach, which shows high accuracy, enables the shear and flexural deformation measurement being stripped from each other in variable depth specimen.

3) For low stirrup ratio specimens, the formation of first critical diagonal cracks and stirrup yielding are the turning points of load-deformation curve. The tested series shows that the shear stiffness will decrease $2 / 3$ after the diagonal cracking, and nearly $90 \%$ after stirrup yielding. Study also shows that raising the concrete strength and stirrup ratio can restrain the development of shear deformation and improve the residual shear stiffness after shear diagonal cracking.

This paper is supported by the Opening Project of Key Laboratory of Concrete and Pre-stressed Concrete Structures of Ministry of Education, Southeast University.

\section{References}

1. A. Tena-Colunga, H. I. Archundia-Aranda, and Ó. M. González-Cuevas, Eng Struct, 30(2), 478 (2008)

2. H. M. Elsanadedy, et al, Mater \& Struct,49(9), 3749 (2016)

3. M. P. Collins, E. C. Bentz, E. G. Sherwood, ACI Struct J, 105(5), 125 (2008)

4. F. Leonhardt, R. Walther, Cem Concr Res, 111, 1 (1964)

5. R. Park, T. Pauley, Reinforced concrete structures, (1975)

6. P.Debernardi, M. Guiglia, \& M. Taliano, Eng Struct, 33(2), 368 (2011)

7. C. Hansapinyo, A. Pimanmas, K. Maekawa, and T. Chaisomphob, J JSCE, Division E , 58, 305 (2003)

8. K. Zheng, Z. Liu, G. Shi, China Civ Eng J , 48(S1), 1 (2015) (in Chinese)

9. ASCE-ACI Committee 445, J Struct Eng, 124(12), 1 (1998)

10. ACI Committee 318 , Building code requirements for structural concrete (ACI318-08), (2008)

11. Z. He, Z.Liu, Z. J. Ma, J Struct Eng, 142(2): 06015003 (2016)

12. American Association of State Highway and Transportation Officials (AASHTO), AASHTO LRFD bridge design specifications, (2012) 